anales de psicología, 2015, vol. 31, nº 2 (mayo), 600-606 http://dx.doi.org/10.6018/analesps.31.2.156391
(C) Copyright 2015: Servicio de Publicaciones de la Universidad de Murcia. Murcia (España) ISSN edición impresa: 0212-9728. ISSN edición web (http://revistas.um.es/analesps): 1695-2294

\title{
Aceptación-rechazo parental y perfiles de victimización y agresión en situaciones de bullying
}

\author{
Benito León-del-Barco ${ }^{1 *}$, Elena Felipe-Castaño ${ }^{2}, \mathrm{M}^{\mathrm{a}}$ Isabel Polo-del-Río y Fernando Fajardo-Bullón ${ }^{1}$ \\ ${ }^{1}$ Facultad de Formación del Profesorado, área de Psicología Evolutiva y de la Educación. Dpto. Psicología y Antropología. UEX. Universidad de Extremadura. \\ ${ }^{2}$ Facultad de Formación del Profesorado, área de PETRA. Dpto. Psicología y Antropología. UEX. Universidad de Extremadura.
}

\begin{abstract}
Resumen: Un área de estudio importante en el acoso escolar continúa siendo el análisis de las causas del fenómeno y de los factores que actuarían como protección/riesgo en los ámbitos culturales, sociales, personales, es colares y familiares. La familia puede ser clave como factor de protección o de riesgo en la aparición de situaciones de violencia escolar. Con este estudio mediante un análisis discriminante pretendemos determinar las relaciones entre los diferentes perfiles de victimización y agresión implicados en la dinámica bullying y la aceptación-rechazo que perciben los participantes de sus padres. La muestra total estaba formada por 700 adolescentes estudiantes, $43 \%$ mujeres y $57 \%$ varones con una media de edad de 13.98 . Hemos utilizado el Cuestionario de Convivencia Escolar (Defensor del Pueblo, 2006) y la Escala de Afecto versión hijos, EA-H (Bersabé, Fuentes y Motrico, 2001). Nuestros resultados confirman que las víctimas se caracterizarían por percibir un mayor afecto y comunicación de sus madres. Los agresores y los agresores/víctimas por percibir rechazo y crítica, especialmente del padre y poco afecto y comunicación de sus madres.

Palabras clave: Bullying; agresores; víctimas; adolescentes; familia; afecto parental; rechazo parental.
\end{abstract}

Title: Parental acceptance-rejection and profiles of victimization and aggression in bullying situations.

Abstract: An important area of study in bullying remains the analysis of the causes of the phenomenon and the factors that act as protective / risk in the cultural, social, personal, school and family. The family may be key protective factor or risk in the occurrence of school violence situations. With this study by discriminant analysis aims to determine the relationships between the different profiles of victimization and aggression dynamics involved in bullying and acceptance-rejection participants perceived their parents. The total sample consisted of 700 adolescent students, $43 \%$ female and $57 \%$ men with a mean age of 13.98 . We have used the Cuestionario de Convivencia Escolar (Defensor del Pueblo, 2006) and Escala de Afecto versión hijos, EA-H (Bersabé, Fuentes y Motrico, 2001). Our results confirm that the victims would be characterized by greater affection and receive communication from their mothers. The attackers and attackers / victims to perceive rejection and criticism, especially from the father and little affection and communication of their mothers.

Key words: Bullying; bullies; victims; adolescence; family; parental affection; parental rejection.

\section{Introducción}

Para Olweus (1983) el maltrato o abuso entre iguales es una conducta de persecución física y/o psicológica que realiza el alumno o alumna contra otro, al que elige como víctima de repetidos ataques. El bullying no es un fenómeno nuevo, pero si es el más estudiado de todos cuantos pueden ocurrir en el contexto escolar, y es el que más daño moral, psicológico y educativo produce entre los escolares (Avilés, 2006). Olweus (2001) describe los distintos roles que se pueden dar en una situación de acoso escolar (agresor, víctima, agresorvíctima y observadores o testigos) como el círculo del bullying y considera que todos los participantes van a verse afectados en un sentido negativo por esta situación. Los roles de víctimas y agresores presentan dimensiones de personalidad diferenciadoras. Cerezo (2001) en una muestra de 315 alumnos de 10 a 15 años encuentra que las víctimas viven sus relaciones interpersonales con un alto grado de timidez que las llevan al retraimiento y al aislamiento social. Entre los rasgos de personalidad, estos alumnos presentan una alta puntuación en ansiedad e introversión. Estévez, Martínez y Musitu (2006) sobre un total de 965 adolescentes con edades comprendidas entre los 11 y 16 años muestran que los adolescentes victimizados en la escuela se perciben a sí mismos más negativamente en los ámbitos social y emocional de la autoestima. En el otro lado, los agresores tienen tendencia hacia la hiperactividad-impulsividad (Farrington, 2005), son extravertidos, líderes, sinceros, manifiestan una

* Dirección para correspondencia [Correspondence address] Benito León del Barco. E-mail: bleon@unex.es considerable asertividad (Cerezo, 2001), alta autoestima en al ámbito social y emocional (Estévez, Martínez y Musitu, 2006) y presentan un alto nivel de psicoticismo (Slee y Rigby, 1993).

La preocupación social que suscita la violencia en los contextos sociales ha dado lugar a numerosas intervenciones y acciones en diferentes países y en muy diferentes ámbitos (Martín, Fernández, Andrés, Del Barrio y Echeita, 2003). Para Benítez y Justicia (2006) en una fase inicial las investigaciones se han centrado junto a la definición del problema, en el análisis epidemiológico de la gravedad del fenómeno en cada contexto. Como resultado final de todas las investigaciones previas ha aumentado el desarrollo e implementación de programas y estrategias de prevención e intervención (Cowie y Olaffson, 2000; Díaz-Aguado, 2005). Un área de estudio importante continua siendo el análisis de las causas del fenómeno (Farrington, 2005) y de los factores que actuarían como protección/riesgo en los ámbitos culturales, sociales, personales, escolares y familiares (Buelga, Cava y Musitu, 2012; Cerezo y Ato, 2010; Gázquez, Cangas, PérezFuentes, Padilla y Cano, 2007; Olweus, 1998; Ortega y Mora-Merchan, 2000; Rigby 2002, Smith, 2005).

La familia puede ser clave como factor de protección o de riesgo en la aparición de situaciones de violencia escolar (Piñero y Cerezo, 2010). Los padres constituyen el principal medio de socialización y desarrollo de toda persona desde muy temprana edad. Es indiscutible el papel de la familia en el desarrollo psicosocial, los padres son la fuerza más poderosa en la vida de sus hijos. Para Rodrigo y Palacios (1998) las concepciones de los padres sobre la educación, las relaciones interpersonales y los estilos de crianza son factores 
determinantes para el desarrollo de los hijos. Los estilos parentales han sido utilizados para explicar los efectos de la socialización familiar sobre la competencia de los hijos. Particularmente, influyente en el estudio de los estilos parentales ha sido la aportación de Baumrind (1971), quién delineó diferentes estilos parentales que hoy son ampliamente conocidos en la literatura científica. Aunque la mayoría de las investigaciones se ajustan al modelo de Maccoby y Martin (1983), que clasifica a los padres en cuatro estilos a partir de dos dimensiones (afecto y control).

Distintos estudios resaltan como el estilo democrático de los padres, caracterizado por una disciplina inductiva, el afecto y el control positivo, juega un papel decisivo en el ajuste social y comportamental de sus hijos y sobre aspectos concretos de su personalidad como es la autoestima (Pons y Berjano, 1997; Torío, Peña e Inda, 2008). Una relación positiva con los padres, basadas en la expresión positiva de los sentimientos, es un factor de protección frente a la victimización en la escuela en adolescentes (Estévez, Murgui, Musitu y Moreno, 2008). Por otro lado, los estilos parentales caracterizados por prácticas restrictivas, punitivas y pobre supervisión están relacionados con el comportamiento violento de los hijos. Para Torío et al. (2008) un estilo parental autoritario tiene repercusiones negativas sobre la socialización porque genera falta de autonomía personal, menor competencia social y baja autoestima. En este sentido, estudios realizados por Llopis y Llopis (2001) y López-Soler, Puerto, López-Pina y Prieto (2009) manifiestan que la percepción del estilo paternal autoritario por parte de los hijos no favorece el ajuste personal y social, pudiendo provocar conductas inadecuadas socialmente como la agresividad hacia los iguales.

Un problema relacionado con la investigación de estilos parentales es que los padres no tienen un estilo de crianza definido. Los estilos parentales no son excluyentes, los padres pueden manifestar un estilo principal con prácticas parentales específicas de otros. Además, el momento evolutivo del niño, su comportamiento y actitudes tendrían un impacto sobre las prácticas parentales, para Palacios (1999) la influencia entre estilos y socialización sería bidireccional. Por otro lado, aunque la mayoría de las investigaciones se ajustan al modelo de dos dimensiones de Maccoby y Martin (1983), existen otras dimensiones como la promoción de la autonomía, la revelación, el humor... que habría que tener en cuenta en un modelo multidimensional de los estilos parentales (Oliva, Parra y Arranz, 2008). Por estos motivos en nuestro trabajo nos hemos centrado en una dimensión y analizaremos la relación entre las conductas parentales de aceptación-rechazo con los perfiles de agresores y víctimas en situaciones de acoso escolar.

Según Rohner (1975) la aceptación rechazo parental implica un continuo, en un extremo estarían los padres que muestran a sus hijos amor y afecto tanto verbal como físicamente. En el otro extremo encontramos padres que sienten aversión hacia sus hijos, les critican y les rechazan. El afecto y la comunicación previenen conductas desajustadas en los adolescentes y estimulan el desarrollo positivo (Mansager y Volk, 2004; Sánchez-Sandoval, 2002). La afectividad positiva de padres sobre hijos constituye un requisito preventivo para la salud mental (Funes, 1984). Por otro lado, investigaciones como las de Repetti, Taylor y Seeman (2002) y Steinberg (2001) confirman que niveles inadecuados de afecto, de apoyo y predominio de la agresión y el rechazo hacia los hijos, se relacionan con la manifestación de problemas conductuales de agresividad, hostilidad y delincuencia. Muris, Meesters, Morren y Moorman (2004) realizaron una investigación con una muestra no clínica de 441 adolescentes en la que encontraron que bajos niveles de calidez emocional y altos niveles de rechazo por parte de los padres correlacionaron con altos niveles de ira y hostilidad entre los adolescentes.

Con este estudio mediante un análisis discriminante pretendemos determinar las relaciones entre los diferentes perfiles de victimización y agresión implicados en la dinámica bullying y la aceptación-rechazo que perciben los participantes de sus padres. ¿Por qué nos interesa la percepción de los hijos sobre la aceptación-rechazo de sus padres? Diversos estudios encuentran baja coincidencia entre las opiniones de los padres e hijos sobre las prácticas parentales (Bernabé, Fuentes y Motrico, 2001; González y Landero, 2012). Los padres suelen tener una percepción propia sobre sus prácticas parentales, a veces sesgada por una deseabilidad social. La percepción de los hijos adolescentes tiene menos sesgo, es más objetiva y puede ser un importante predictor de sus respuestas, más que la de los padres.

Asimismo, analizaremos qué variables de la aceptaciónrechazo parental presentan un mayor poder de discriminación o cuantifican mejor las diferencias entre los diferentes perfiles implicados en el bullying. En este objetivo nos planteamos dos hipótesis. Primera: la variable afecto y comunicación de la madre tendrá una mayor capacidad para discriminar el perfil víctima y segunda: la variable crítica y rechazo del padre tendrá una mayor capacidad para discriminar el perfil agresor y agresor/víctima.

\section{Método}

La muestra total estaba formada por 700 adolescentes estudiantes, $43 \%$ mujeres y $57 \%$ varones con una media de edad de 13.98 (DT = 1.38). El número de participantes se determinó a partir del número de alumnos matriculados en Educación Secundaria Obligatoria (ESO) en centros públicos y concertados de la Comunidad de Extremadura durante el curso 2008-2009, considerando un error muestral de 3\% y un nivel de confianza de $95.5 \%$. La selección de la muestra se realizó mediante un muestreo polietápico por conglomerados y selección aleatoria de los grupos en los centros que disponían de varias líneas en los cursos $1^{\circ}, 2^{\circ}, 3^{\circ}$ y $4^{\circ}$ de la ESO. El muestreo por conglomerados se llevó a cabo seleccionando al azar cuatro centros. En cuanto a la distribución por curso de nuestros participantes, 190 alumnos eran de $1^{\circ}$ de ESO, 177 de $2^{\circ}$ de ESO, 171 de $3^{\circ}$ y 162 de $4^{\circ}$ de ESO. 


\section{Instrumentos}

Cuestionario sociodemográfico elaborado de forma específica para la investigación y que contenía cuestiones relativas a edad, género, nivel educativo y datos sociodemográficos y socioeconómicos de los padres.

Cuestionario de Convivencia Escolar (Defensor del Pueblo, 2006). Se utilizaron dos escalas en las que se pedía que contestaran según un formato tipo Likert de cuatro intervalos, que van de 1 "nunca" a 4 "siempre", si habían vivido desde que empezó el curso 13 situaciones de acoso escolar desde la perspectiva de víctima y agresor/a. Las situaciones son las siguientes: Ignorarle, no dejarle participar, insultarle, ponerle motes que le ofenden o ridiculizan, hablar mal de él o ella, esconderle cosas, romperle cosas, robarle, pegarle, amenazarle para meterle miedo, acosarle sexualmente, obligarle a hacer cosas que no quiere hacer con amenazas y amenazarle con armas. El análisis de consistencia interna de las dos escalas, calculado mediante el alfa de Cronbach, nos indica valores de .84 para la escala de Víctimas y .89 para Agresores. A continuación, presentamos los resultados de un análisis factorial exploratorio y confirmatorio para ambas escalas.

Escala de Víctimas: los resultados de la prueba KMO (.775) y Bartlett $\left(\chi^{2}=984.851\right.$ y $\left.p<.000\right)$, indican la pertinencia de realizar un análisis factorial. Sometimos los datos a un análisis factorial con rotación Oblimin, encontrando una estructura de 3 factores que explican el 55\% de la varianza total. El primer factor formado por 7 ítems, "Victima de agresiones físicas y amenazas", explica el $36.5 \%$ de la varianza y se refiere a conductas como: me roban, me obligan a hacer cosas, me rompen cosas, me pegan, me amenazan para meter miedo... El segundo factor de 5 ítems, "Victima de agresiones verbales y de exclusión social," explica el 10\% de la varianza y se refiere a conductas como: me insultan, hablan mal de mí, me ponen motes, me ignoran y no me dejan participar. El último factor de un ítem explica el $8.5 \%$ de la varianza y se denomina "Victima de acoso sexual".

Por otro lado, para analizar el ajuste de la estructura factorial teorizada a nuestros datos realizamos un análisis factorial confirmatorio, utilizando el método de Máxima Verosimilitud (Jöreskog y Sörbom, 1996). Todos los indicadores, a excepción de CMIN/DF, obtienen valores alejados de los límites aceptados y nos permiten afirmar que el modelo no presenta un buen ajuste $\left(\chi^{2}=225.701, p<.000 ; C M I N / d f=\right.$ 3.583; $C F I=.821 ; T L I=.742 ; R M S E A=.082)$. Para tratar de verificar la estructura factorial teorizada a partir de los diferentes ítems hemos utilizado el método de codificación de efectos propuesto por Litte, Slegers y Card (2006), encontrando los siguientes valores en las cargas factoriales: Factor (Victima de agresiones físicas y amenazas), valores que oscilan entre $\lambda=.300$ y $\lambda=.716$ con una media de $\lambda=.540$; Factor (Victima de agresiones verbales $y$ de exclusión social), valores entre $\lambda=.454$ y $\lambda=.589$ con una media de $\lambda=.512$ y Factor (Victima de acoso sexual), con un valor de $\lambda=.808$.

Escala de Agresores: los resultados de la prueba KMO (.872) y Bartlett $\left(\chi^{2}=2628.996\right.$ y $\left.p<.000\right)$, indican que tiene sentido realizar el análisis factorial. Después del análisis con rotación Oblimin encontramos tres factores que explican el $65 \%$ de la varianza total. El primer factor formado por 7 ítems al que llamaremos "Agresiones físicas y amenazas" explica el $31 \%$ de la varianza y se refiere a conductas como: robo, obligo a hacer cosas, rompo cosas, pego, amenazo para meter miedo... El segundo factor de 5 ítems, "Agresiones verbales $y$ de exclusión social", explica el $25 \%$ de la varianza y se refiere a conductas como: insulto, hablo mal, pongo motes, ignoro y no dejo participar. El tercer factor formado por un solo ítem explica el 9\% de la varianza y lo hemos llamado "Acoso sexual".

Por último, para analizar el ajuste de la estructura factorial teorizada a nuestros datos realizamos un análisis factorial confirmatorio, utilizando el método de Máxima Verosimilitud. Todos los indicadores para determinar si el modelo se ajusta adecuadamente a los datos, obtienen valores alejados de los límites aceptados y nos permiten afirmar que el modelo no presenta un buen ajuste $\left(\chi^{2}=450.167, p<.000\right.$; $\mathrm{CMIN} / \mathrm{DF}=7.146 ; C F I=.850 ; T L I=.783 ; \mathrm{RMSE} A=.126)$. Por otro lado, utilizando en la estimación el método de codificación de efectos, hemos encontrado los siguientes valores en las cargas factoriales: Factor (Agresiones físicas y amenazas), valores que oscilan entre $\lambda=.538$ y $\lambda=.876$ con una media de $\lambda=.742$; Factor (Agresiones verbales y de exclusión social), valores entre $\lambda=.596$ y $\lambda=.808$ con una media de $\lambda=.685$ y Factor (Acoso sexual), con un valor de $\lambda=.940$.

Los análisis confirmatorios de ambas escalas verifican que el modelo encontrado en el análisis factorial exploratorio no presenta un buen ajuste. Las significativas y altas correlaciones en ambas escalas entre los factores, a excepción del Factor (Acoso sexual), ponen en duda la multidimensionalidad de las escalas, a pesar de que los valores en las cargas factoriales indican que los factores están en principio bien definidos a través de los diferentes ítems. Los resultados del análisis confirmatorio avalan las dificultades que supondría investigar utilizando factores en las Escalas Víctima y Agresor del Cuestionario de Convivencia Escolar (Defensor del Pueblo, 2006). Este es el principal argumento por el cual en nuestra investigación hemos utilizado las puntuaciones totales obtenidas por el sumatorio de las respuestas a los 13 ítems que constituyen cada escala.

Escala de Afecto versión hijos, EA-H (Bersabé, Fuentes y Motrico, 2001). Se compone de dos factores, cada uno consta de 10 ítems que se presentan en formato Likert con cinco grados de frecuencia que representan un continuo que va desde Nunca hasta Siempre.

El primer factor llamado Afecto-Comunicación evalúa la percepción que tienen los hijos del afecto, el interés y comunicación que manifiestan sus padres (Padre-Madre) hacia ellos: "Me consuela cuando estoy triste", "Me acepta tal y como soy", "Es cariñoso/a conmigo". Presenta un alfa de Cronbach para la modalidad padre de .90 y .87 para la modalidad madre. El segundo factor llamado Crítica-Rechazo evalúa la crítica, el rechazo y la falta de confianza de los padres $(\mathrm{Pa}-$ dre-Madre) hacia sus hijos: "Lo que hago le parece mal", 
"Está a disgusto cuando yo estoy en casa", "Le gustaría que fuera diferente". Presenta un alfa de Cronbach para la modalidad padre de .83 y de .81 para la modalidad madre.

\section{Procedimiento}

El procedimiento seguido para la obtención de datos fue mediante la administración de los cuestionarios por grupo de clase. En primer lugar, nos pusimos en contacto con los centros educativos para explicarles los objetivos del estudio y solicitarles autorización para la cumplimentación de los cuestionarios. Seguimos las directrices éticas de la American Psychological Association (APA, 2009) con respecto al consentimiento informado de los padres, debido a la minoría de edad de los participantes. Asimismo, se aseguró el anonimato en las respuestas, la confidencialidad de los datos obtenidos y su utilización exclusiva para fines de investigación. La administración de los cuestionarios se hizo en horario escolar, ocupaba en torno a 40 minutos en un clima adecuado y sin distracciones. Los análisis estadísticos de los datos recogidos se realizaron utilizando el programa estadístico SPSS 19.0 para PC.

\section{Resultados}

En primer lugar se seleccionaron diferentes subgrupos de la muestra final en función de los roles víctima y agresor asumidos por los participantes. Para ello se utilizaron las puntuaciones en las dos escalas del Cuestionario del Defensor del Pueblo (2006) en los que se preguntaba a los sujetos por su participación, según los roles víctima y agresor descritos por Olweus (1993), en diversas situaciones de bullying. En total son 13 situaciones distintas que se han enumerado en el apartado de instrumentos y a las que los sujetos debían responder en función de si habían sufrido (víctima) o provocado (agresor) esa situación. Para calcular la frecuencia de participación en esa situación se realizó un sumatorio de las respuestas a esos 13 ítems, de manera que obtuvimos dos nuevas variables denominadas intensidad de la experimentación de la situación de víctima y agresor. En estas variables se obtuvieron valores de entre 13 y 52 , indicando el valor de 13 , ausencia de experiencias y el valor 52 gran presencia de experiencias en todas las situaciones de bullying.

Una vez obtenidas estas variables se calculó el valor de percentil 75 para seleccionar aquellos participantes que hubiesen experimentado un mayor número e intensidad de las situaciones. Las puntuaciones descriptivas y de percentiles para cada uno de los roles fueron los siguientes: agresores $(M=15.65, D T=3.98$, percentil 75: 16), víctimas $(M=14.53$, $D T=2.55, P 75: 15)$. Seleccionamos subgrupos dentro de la muestra final y encontramos que había sujetos que podíamos incluir en varias combinaciones posibles dentro de estos roles, de forma que finalmente se establecieron los subgrupos de roles: agresores $(n=117)$, víctimas $(n=90)$ y agresor/víctima $(n=88)$.

En la Tabla 1 podemos ver las medias y desviaciones típicas de cada uno de los roles de participación en la dinámica bullying para los cuatro factores de la EA-H. Las víctimas obtienen puntuaciones medias más altas en afecto del padre, como de la madre. Por el contrario, los agresores y agresores/víctimas obtienen puntuaciones medias más altas en rechazo de los padres.

Tabla 1. Medias y desviaciones típicas factores EA-H en función de los diferentes perfiles bullying

\begin{tabular}{|c|c|c|c|c|}
\hline & \multicolumn{4}{|c|}{ Escala de Afecto versión hijos, EA-H } \\
\hline & Afecto Padre $M(D T)$ & Rechazo Padre $M(D T)$ & Afecto Madre $M(D T)$ & Rechazo Madre $M(D T)$ \\
\hline Víctima & $37.96(9.12)$ & $17.24(6.66)$ & $42.13(6.80)$ & $17.22(6.30)$ \\
\hline Agresor & $34.56(9.87)$ & $20.22(8.13)$ & $38.74(8.07)$ & $19.09(6.66)$ \\
\hline Agresor/Víctima & $35.74(9.50)$ & $20.37(7.85)$ & $39.09(7.10)$ & $20.32(6.85)$ \\
\hline
\end{tabular}

A continuación, se examinó la posible existencia de diferencias entre las medias de los tres grupos de roles de participación en el bullying en cuanto a las puntuaciones en los factores de la EA-H. Para ello se realizó un análisis de la varianza (ANOVA), encontrando diferencias significativas entre los tres grupos para todos los factores de la EA-H. Para el factor Afecto Padre (Wilks $\lambda=.978, F=2.701, p<.050$ ); Rechazo Padre (Wilks $\lambda=.967, F=4.138, p<.017$ ); Afecto Madre (Wilks $\lambda=.961, F=5.000 p<.007$ ); Rechazo Madre (Wilks $\lambda=.967, F=4.176, p<.016$ ).

Una vez demostradas la existencia de diferencias entre la medias de los tres grupos de roles de participación en el bullying, analizaremos mediante el análisis discriminante, qué factores de la EA-H explican en mayor medida esas diferencias. El análisis discriminante nos permite determinar la existencia de diferencias significativas entre los diferentes roles de participación en el bullying (agresor, víctima, y agre- sor/víctima) en relación a un conjunto de variables, en nuestro caso la aceptación-rechazo parental (Afecto y comunicación del padre, rechazo y crítica del padre, afecto y comunicación de la madre, rechazo y crítica de la madre). Igualmente, mediante el análisis discriminante pretendemos clasificar a los participantes y asignarlos a cada uno de los roles en el bullying en función del resultado de la combinación lineal del conjunto de variables independientes. En nuestro estudio hemos utilizado como variables independientes y predictoras los cuatro factores de la EA-H. Como variables dependientes los diferentes roles de participación en el bullying.

Es necesario para poder realizar el análisis discriminante que se cumplan los supuestos de linealidad, que todas las variables observadas incluidas en el modelo sigan una distribución normal y la igualdad de las varianzas-covarianzas. Los gráficos de dispersión de los residuos realizados reflejaron que existe linealidad entre las variables estimadas. Someti- 
mos los datos a la prueba de Kolmogorov-Smirnov para analizar la distribución normal, encontrando $p<.05$ y normalidad para todas las variables. Por otro lado, el valor 24.413 obtenido en test $M$ de Box y $p=.250$ demuestra la igualdad de las matrices de las covarianzas de los grupos.

En la Tabla 2 se muestra la matriz de estructura que se crea en el análisis discriminante. El número máximo de funciones discriminantes o combinaciones lineales es igual a una unidad menos que el número de grupos asignados a la variable dependiente. ¿Qué función tiene un mayor poder de discriminación y utilizaremos para interpretar los datos? El análisis de las funciones discriminantes nos indica que la Función 1 es la que presenta un mayor poder de discriminación entre los tres grupos de roles.

Tabla 2. Matriz de la estructura. Variables ordenadas por el tamaño de la correlación con la función discriminante.

\begin{tabular}{lcc}
\hline & \multicolumn{2}{c}{ Funciones } \\
\cline { 2 - 3 } & Función 1 & Función 2 \\
\hline Afecto y Comunicación Madre & $-.834^{*}$ & .194 \\
Rechazo y Crítica Padre & $.763^{*}$ & .045 \\
Rechazo y Crítica Madre & $.701^{*}$ & .644 \\
Afecto y Comunicación Padre & $-.575^{*}$ & .461 \\
\hline * Mayor correlación absoluta entre cada variable y la función discriminante
\end{tabular}

La Función 1 explica un porcentaje de varianza muy superior al resto de funciones, muestra una mayor correlación canónica y distancia entre los grupos discriminados (Lambda de Wilks más cercano a 0). Además, el análisis de $\chi^{2}$ presenta el más elevado nivel de significación. Función 1 (\% de varian$\mathrm{za}=81.1$, correlación canónica $=.234$, Wilks $\lambda: .933, \chi^{2}$ $=16.979, g l=8, p<.030)$; Función $2(\%$ de varianza $=18.9$, correlación canónica $=.115$, Wilks $\lambda: .987, \chi^{2}=3.268, g l=3, p$ $<.352)$. Por tanto, según la Función 1 el factor que mayor capacidad predictiva tiene es Afecto Comunicación Madre (.834). Le siguen el factor Rechazo y Crítica Padre (.763) y el factor Rechazo y Crítica Madre (.701). Para poder interpretar la relación de cada factor con los diferentes roles necesitamos conocer las medias y especialmente el signo de las funciones en los centroides de los grupos. Para el rol Víctima= .367 , para el rol Agresor $=.152$ y para el Agresor/Víctima $=$ .161. En definitiva, el grupo de víctimas discrimina del resto de roles, pero apenas hay diferencias entre el grupo de agresores y agresores/víctimas. Las víctimas se caracterizarían por percibir un mayor afecto y comunicación de sus madres. Los agresores y los agresores/víctimas por percibir rechazo y crítica, especialmente del padre y poco afecto y comunicación de sus madres.

Por último, en la Tabla 3 observamos que la función canónica discriminante obtenida permite clasificar correctamente el $56.8 \%$ de las víctimas, el $34.7 \%$ de los agresores y el $28.9 \%$ de los agresores/víctimas. Ganancias medias en la predicción superiores al 33\% que acertaríamos por azar en las 3 categorías de roles (Víctima, Agresor y Agresor/víctima).
Tabla 3. Resultados de la clasificación empleando la función discriminante.

\begin{tabular}{ccccc}
\hline & \multicolumn{3}{c}{ Grupo de pertenencia pronosticado } \\
\cline { 2 - 5 } & Víctima & Agresor & Agresor/Víctima \\
\hline Recuento Víctima & 42 & 18 & 14 \\
& Agresor & 38 & 34 & 26 \\
& Agresor/Víctima & 33 & 21 & 22 \\
\hline$\%$ & Víctima & 56.8 & 24.3 & 18.9 \\
& Agresor & 38.8 & 34.7 & 26.5 \\
& Agresor/Víctima & 43.4 & 27.6 & 28.9
\end{tabular}

Clasificados correctamente el $40 \%$ de los casos agrupados originales

\section{Discusión}

El objetivo principal de este trabajo es analizar qué variables de la aceptación-rechazo parental presentan un mayor poder de discriminación o cuantifican mejor las diferencias entre los diferentes perfiles de victimización y agresión implicados en el bullying. Los resultados obtenidos ponen de manifiesto la existencia de diferencias entre las medias de los tres grupos de roles de participación en el bullying según las puntuaciones en los factores de la EA-H y que los dos factores que mejor cuantifican las diferencias entre los perfiles de victimización y agresión son por este orden el Afecto y Comunicación Madre y Rechazo Crítica Padre. En este sentido encontramos que el afecto y comunicación que perciben los hijos de sus madres se relaciona con la victimización, mientras que, principalmente, el rechazo, la aversión y la crítica que perciben los hijos, especialmente del padre, constituirían un factor de riesgo en la agresión unido a la falta de afecto y comunicación de la madre.

Respecto al hecho de que el afecto y comunicación que perciben los hijos de sus madres se relaciona con la victimización, investigaciones realizadas por Olweus (1978) indican que los chicos víctimas de agresiones tienen con sus padres, especialmente con sus madres, un contacto más estrecho y unas relaciones más positivas. Este contacto más estrecho y comunicación vendría determinado por las características de las víctimas: ansiedad, reacción sumisa y, en el caso de los chicos, debilidad física. Olweus (1993) nos habla de desviaciones externas en las víctimas, refiriéndose a cuestiones como: tener gafas, ser obeso o débil, dificultades del habla... Farrington (1993) señala como característica de las víctimas la debilidad física y Rigby (2002) indica problemas de salud.

Respecto a la primera hipótesis nos preguntamos ¿por qué el factor afecto comunicación madre cuantifica mejor las diferencias cuando se relaciona con la victimización que el factor afecto comunicación padre? Algunas investigaciones encuentran que las mujeres tienen una mayor sensibilidad que los hombres. En general las mujeres son más expresivas que los hombres, autores como Lafferty (2004) y Tapia y Marsh (2006) entre otros, demostraron en sus investigaciones que las mujeres son más perceptivas, muestran mayor empatía y reconocen mejor las emociones ajenas, esto explicaría una interacción afectiva más positiva y una mayor protección hacia los hijos, especialmente con los hijos caracterizados por una cierta prevención y sensibilidad. Otras investigaciones ponen de manifiesto la mayor implicación de las 
madres frente a los padres en las prácticas de crianza, así Laible y Carlo (2004) comprueban que las madres son más afectuosas y favorecen más la autonomía de los hijos. Por otro lado, Tur, Mestre, Samper y Malonda (2012) con una muestra de 2.788 alumnos de 10-15 años confirman que las madres mantienen una mayor influencia (autonomía y amor) sobre los hijos, independientemente de sexo de los mismos.

Nuestros resultados más relevantes demuestran que el rechazo, la aversión y la crítica que perciben los hijos, especialmente del padre, constituyen un factor de riesgo en la agresión unido a la falta de afecto y comunicación de la madre. Estos resultados coinciden con los estudios de Repetti et al. (2002) que relacionan los estilos parentales caracterizados por un predominio de la agresión y el rechazo hacia los hijos con la manifestación de problemas conductuales de agresividad, hostilidad y delincuencia. Numerosos trabajos destacan como una actitud emotiva negativa caracterizada por carencia de afecto y comunicación incrementa el riesgo de manifestar conductas agresivas (Mestre y Tur, 2007; Loeber y Stouthamer-Loeber, 1986; Olweus, 1980; Tur, Mestre y del Barrio, 2004; Tur, Mestre, Samper y Malonda, 2012)

Con relación a la segunda hipótesis nos planteamos, ¿por qué el factor rechazo crítica padre cuantifica mejor las diferencias en los roles de agresión y agresión/víctima que el factor rechazo crítica madre? Las investigaciones ponen de manifiesto que los varones perciben más rechazo y crítica de su padre que de su madre. Motrico, Fuentes y Bersabé (2001) concluyen que los chicos tienen más conflictos con sus padres que las chicas. Tampoco debemos olvidar que son los chicos los que se ven implicados con mayor frecuencia en el fenómeno bullying, tanto como agresores y víctimas (Cerezo y Ato, 2010; Solberg y Olweus, 2003; Tapper y Boulton, 2004), a excepción de la maledicencia más asociada a las chicas (Defensor del Pueblo, 2006; León, Felipe, Gómez y López, 2011; León, Felipe, Fajardo, Gómez, 2012). Para Sánchez y Cerezo (2010) el sexo es un factor de riesgo para la implicación en bullying, siendo este fenómeno preferentemente masculino.

También, nuestros datos nos permiten concluir que los factores utilizados como predictores resultan de utilidad para clasificar el grupo de víctimas pero de baja eficacia para el grupo de agresores y agresores/víctimas. Griffin y Gross (2004) identifican la figura de agresor/víctima con un grupo de víctimas que en función de las circunstancias asumen el rol de víctima o de agresor. Para Olweus (1993) los agreso-

\section{Referencias}

American Psychological Association. (2009). Publication Manual of the American Psychological Association, Sixth Edition.

Avilés, J. M. (2006). Bullying: El maltrato entre iguales. Agresores, victimas y testigos en la escuela. Salamanca: Amarú Editorial.

Baumrind, D. (1971). Current patterns of parental authority. Developmental Psychology Monograph, 4, 1-103.

Benítez, J. L. y Justicia, F. (2006). El maltrato entre iguales: descripción del fenómeno. Electronic Journal of Research in Educational Psychology, 9 4(2), 151-170.

Bersabé, Fuentes y Motrico (2001). Análisis Psicométrico de dos escalas para evaluar estilos educativos parentales. Psicothema, 13, 678-684. res/víctimas constituyen un grupo de menor tamaño muy agresivo, se enfrentan tanto a los agresores como a las víctimas pasivas y les denomina víctimas provocativas. En definitiva, el grupo de agresores y agresores/víctimas se caracterizan por la agresión y las diferencias entre unos y otros están muy difuminadas. Sería interesante en futuras investigaciones, mediante un análisis discriminante predictivo, utilizar como variable dependiente sólo dos grupos de roles el de víctima por un lado y el de agresores por otro.

Queremos indicar que el estudio presenta varias limitaciones, como son la utilización de autoinformes por los participantes tanto para la evaluación de las situaciones de bullying como de la aceptación-rechazo parental. Creemos que sería importante contar con otros informantes además de los involucrados directamente. Consideramos que los profesores se encuentran en una situación privilegiada para analizar las conductas de victimización y agresión ya que pueden observarlas y evaluarlas en la situación diaria de clase y pueden establecer comparaciones con otros niños y niñas del mismo nivel de desarrollo. Asimismo, dentro de un modelo bidireccional de las relaciones padres-hijos sería conveniente evaluar la aceptación-rechazo desde el punto de vista de los padres. Por otro lado, las diferencias en el número de casos en cada uno de los roles debe llevarnos a considerar los resultados de forma cautelosa hasta poder ampliar este número (agresores $n=117$, víctimas $n=90 \mathrm{y}$ agresor/víctima $n=88)$.

Finalmente, consideramos que es necesario implementar programas de prevención del acoso escolar en todos los centros educativos y coincidimos con Díaz-Aguado (2005) en la importancia de erradicar las situaciones de exclusión desde las primeras etapas educativas. Asimismo, sería conveniente el diseño de programas de intervención y formación con las familias para conseguir mejorar las relaciones entre padres e hijos (Tur, Mestre, Samper y Malonda, 2012). Creemos, también, en una formación de padres orientada a que éstos descubran que el origen de los problemas de sus hijos puede encontrarse en su propio comportamiento. Esta formación incrementa la conciencia de los padres y la utilización de sus aptitudes y competencias. Para Cataldo (1991) la formación de padres forma parte de la educación de los niños y es un método para promover su desarrollo, mejora las relaciones familiares y aumenta el sentimiento de satisfacción y competencia de los padres respecto a sus tareas y responsabilidades como padres y madres.
Bowers, L., Smith, P. K. y Binney, V. (1992). Cohesion and power in the families of children involved in bully/victim problems at school. Journal of Family Therapy, 14(4), 371-387.

Buelga, S., Cava, M. J. y Musitu, G. (2012). Reputación social, ajuste psicosocial y victimización entre adolescentes en el contexto escolar. Anales de Psicología, 28(1), 180-187.

Cataldo, C. Z. (1991). Aprendiendo a ser padres: conceptos y contenidos para el diseño de programas de formación de padres. Madrid: Visor. 
Cerezo, F. (2001). Variables de personalidad asociadas a la dinámica bullying (agresores versus víctimas) en niños y niñas de 10 a 15 años. Anales de Psicología, 17(1), 37-43.

Cerezo, F. y Ato, M. (2010). Social status, gender, classroom climate and bullying among adolescents pupils. Anales de Psicología, 26(1), 137-144.

Cowie, H. y Olafsson, R. (2000). The role of peer support in helping the victims of bullying in a school with high levels of aggression. School Psychology International, 21(1), 79-95.

Defensor del Pueblo (2006). Violencia escolar: el maltrato entre iguales en la Educación Secundaria Obligatoria (1999-2006). Madrid: Publicaciones del Defensor del Pueblo.

Díaz-Aguado, M. J. (2005). La violencia entre iguales en la adolescencia y su prevención desde la escuela. Psicothema, 17, 549-558.

Estévez, E., Martínez, B. y Musitu, G. (2006). La autoestima en adolescentes agresores y víctimas en la escuela: la perspectiva multidimensional. Intervención Psicosocial, 15(2), 223-233.

Estévez, E., Murgui, G., Musitu, G. y Moreno, D. (2008). Adolescent aggression: effects of gender and family and school environments. Journal of Adolescence, $31,433-450$.

Farrington, D. (2005). Childhood origins of antisocial behavior. Clinical Psychology and Psychotherapy, 12, 177-190.

Farrington, D. P. (1993). Understanding and preventing bullying. Crime and justice. Review of Research, 17, 381-458.

Funes, J. (1984). La nueva delincuencia juvenil. Barcelona: Paidós.

Gázquez, J. J., Cangas, A. J., Pérez-Fuentes, M. C., Padilla, D. y Cano, A. (2007). Percepción de la violencia escolar por parte de los familiares: un estudio comparativo en cuatro países europeos. Internacional Journal of Clinical and Health Psychology, 7, 93-105.

González, M. y Landero, R. (2012). Diferencias en la percepción de estilos parentales entre jóvenes y adultos de la misma familia. Summa Psicológica UST, 9(1), 53-64.

Griffin, R. y Gross A. (2004). Chidhood bullying: current empirical findings and future directions for research. Aggressive and violent behavior, 9, 379-400

Jöreskog, K. G. y Sörbom, D. (1996). Lisrel 8: User's reference guide. Chicago: SSI Inc.

Lafferty, J (2004). The relationships betweengender, empathy and aggressive behavious among early adolescents. Dissertation Abstracts International: Section B: The Sciences and Engineering, 64, 6377B.

Laible, D. J. y Carlo, G. (2004). The differential relations of maternal and paternal support and control to adolescent social competence, self-worth and sympathy. Journal of Adolescent Research, 19, 759-782.

León, B., Felipe, E., Fajardo, F. y Gómez, T. (2012). Cyberbullying en una muestra de estudiantes de Educación Secundaria: variables moduladoras y redes sociales. Electronic Journal of Research in Educational Psychology, 10(2), 771-778.

León, B., Felipe, E., Gómez, T. y López, V. (2011). Acoso escolar en la Comunidad de Extremadura vs. Informe Español del Defensor del Pueblo 2006. Electronic Journal of Research in Educational Psychology, 9(2), 565-586.

Litte, T. D., Slegers, D. W. y Card, N. A. (2006). A non-arbitrary method of identifying and scaling latent variables in SEM and MACS models. Structural Equation Modeling, 13, 59-72.

Llopis, D. y Llopis, R. (2001). Estilos educativos y relaciones sociales. En Convergencias y Divergencias en la Sociedad Global. Comunicación presentada en VII Congreso Español de Sociologia. Salamanca.

Loeber, R. y Stouthamer-Loeber, M. (1986). Family factors as correlates and predictors of conduct problems and juvenile delinquecy. Crime and Justice. Revien of Research, 7, 29-149.

López-Soler, C., Puerto, J. C., López-Pina, J. A. y Prieto, M. (2009). Percepción de los estilos educativos parentales e inadaptación en menores pediátricos. Anales de Psicología, 25, 70-77.

Maccoby, E. E. y Martin, J. A. (1983). Socialization in the context of the family: Parent-child interaction. En E. M. Hetherington y P. H. MUSSEN (Eds.), Handbook of Child Psychology, Vol. IV: Socialization, Personality, and Social Development (pp.1-101). New York: Wiley.

Mansager, E. y Volk, R. (2004). Parent's prism. Three dimensions of effective parenting. Journal of Individual Psychology, 60(3), 277-293.

Martín, E., Fernández, I., Andrés, S., Del Barrio, C. y Echeita, G. (2003). La intervención para la mejora de la convivencia en los centros educativos: modelos y ámbitos. Infancia y Aprendizaje, 26, 79-95.

Mestre, M. V. y Tur, A. M. (2007). Estilos de crianza en la adolescencia y su relación con el comportamiento prosocial. Revista Latinoamericana de Psicología, 39(2), 211-225.
Motrico, E., Fuentes, M. J. y Bersabé, R. (2001). Discrepancias en la percepción de los conflictos entre padres e hijos/as a lo largo de la adolescencia. Anales de Psicologia, 17, 1-13.

Muris, P., Meesters, C., Morren, M., y Moorman, L. (2004). Anger and hostility in adolescents: relationship with self-reported attachment style and perceived parental rearing styles. Journal of Psychosomatic Research, 57, 257-64.

Oliva, A., Parra, A. y Arranz, E. (2008). Estilos relacionales parentales y ajuste adolescente. Infancia y Aprendizaje, 31(1), 93-106.

Olweus, D. (1980). Familial and temperamental determinants of aggressive behaviour in adolescent boys: A causal analysis. Development Psychology, 16, 644 660

Olweus, D (1983). Low school achievement and agressive behaviour in adolescent boys. En D. Magnusson y V. Allen (Eds.), Human developrnent. An interactional perspective (pp. 353-365). Nueva York: Academic Press.

Olweus, D, (1993). Bullying at school: What we know and what we can do. Oxford: Blackwells. Trad. cast. Conductas de acoso y amenaza entre escolares. Madrid: Morata, 1998.

Olweus, D. (1978). Agression in the schools: Bullies and whipping boys. Washington, D.C.: Hemisphere.

Olweus, D. (2001). Peer harassment: a critical analysis and some important issues. En J. Juvonen y S. Graham (Eds.), Peer harassment in school: the plight of the vulnerable and victimized, (pp. 3-20). New York: Guilford Press.

Ortega, R. y Mora-Merchán, J. A. (2000). Violencia escolar: mito o realidad. Sevilla: Mergablum.

Palacios, J. (1999). La familia y su papel en el desarrollo afectivo y social. En I. Etxebarría, M. J. Fuentes, F. López y M. J. Ortiz (Coords.), Desarrollo Afectivo y Social (pp. 267-284). Madrid: Pirámide.

Piñero, E. y Cerezo, F. (2010). Las buenas relaciones entre hermanos como factor de protección de dinámica bullying en estudiantes de Educación Secundaria. En J. J. Gázquez y M. C. Pérez (Eds.), Investigación en convivencia escolar: variables relacionadas (pp. 415-420). Granada: GEU.

Pons, J., y Berjano, E. (1997). Análisis de los estilos parentales de socialización asociados al abuso de alcohol en adolescentes. Psicothema, 9, 609-617.

Repetti, R. L., Taylor, S. E. y Seeman, T. E. (2002). Risky families: family social environments and the mental and physical health of offspring. Psychological Bulletin, 128, 330-366.

Rigby, K. (2002). New perspectives on bullying. London: JK Publishers.

Rodrigo, M. J. y Palacios, J. (1998). Conceptos y dimensiones en el análisis evolutivo-educativo de la familia. En M. J . Rodrigo y J. Palacios (Coords.), Familia y Desarrollo Humano (pp. 45-70). Madrid: Alianza.

Rohner, R. P. (1975). They love me, they love me not: a world wide study of the effects of parental acceptance-rejection. New Haven, CT: HRAF.

Sánchez, C. y Cerezo, F. (2010). Variables personales y sociales relacionadas con la dinámica bullying en escolares de Educación Primaria. Electronic Journal of Research in Educational Psychology, 8 (3), 1015-1032.

Sánchez-Sandoval, Y. (2002). El ajuste de niños y niñas y su vida familiar: Un estudio longitudinal. Tesis doctoral. Universidad de Sevilla.

Slee, P. y Rigby, K. (1993). The relationship of Eysenck`s personality factors and self-esteem in schoolboys. Personality and Individual Differences, 14(2), 371-373.

Smith, P. K. (2005). Violencia escolar y acoso: factores de riesgo familiars. En J. Sanmartín (Coord.), Violencia y Escuela (pp.59-76).Valencia: Centro Reina Sofía.

Solberg, M. E. y Olweus, D. (2003). Prevalence estimation of school bullying with the Olweus Bully/Victim questionnaire. Aggressive Behavior, 29(3), 239268.

Steinberg, L. (2001). We know some things: parent-adolescent relationships in retrospect and propect. Journal of Research on Adolescence, 11, 1-19.

Tapia, M. y Marsh, G. E. (2006). The effects of sex and grade-point average on emocional intelligence. Psicothema, 18, 108-111.

Tapper, K. y Boulton, M. J. (2004). Sex differences in levels of physical, verbal, and indirect aggression in spanish adolescents. Aggessive Behavior, 30(2), 123 145

Torío, S., Peña, J. V. e Inda, M. (2008). Estilos de educación familiar. Psicothema, 20 (1), 62-70

Tur, A. M., Mestre, M. V. y del Barrio, V. (2004). Factores moduladores de conducta agresiva y prosocial. El efecto de los hábitos de crianza en la conducta del adolescente. Ansiedad y Estrés, 1,78-88.

Tur, A. M., Mestre, M. V., Samper, P. y Malonda, E. (2012). Crianza y agresividad de los menores: ¿Es diferente la influencia del padre y de la madre?. Psicothema, 24(2), 284-298.

(Articulo recibido: 17-07-2012; revisado: 04-02-2014; aceptado: 10-03-2014) 